\section{SAT0115 FLARING OF RHEUMATOID ARTHRITIS DUE TO TAPERING OF DMARDS: HOW DOES IT AFFECT PATIENTS' WELL-BEING? A STUDY ON PATIENT REPORTED OUTCOMES}

Elise van Mulligen ${ }^{1}$, Angelique Weel ${ }^{1,2}$, Martijn Kuijper $^{2}$, Johanna Hazes ${ }^{1}$, Annette van der Helm - van Mil ${ }^{1,3}$, Pascal de Jong ${ }^{1}{ }^{1}$ Erasmus MC, Rheumatology, Rotterdam, Netherlands; ${ }^{2}$ Maasstad Hospital, Rheumatology, Rotterdam, Netherlands; ${ }^{3}$ Leiden University Medical Center (LUMC), Rheumatology, Leiden, Netherlands

Background: Previous studies have shown that it is possible to taper DMARDs in RA patients with an inactive disease, but this is accompanied with a higher chance of disease flares. Current recommendations advise to taper DMARDs from a clinical viewpoint. However, data on the feasibility of tapering DMARDs from a patient's perspective are sparse. Objectives: To determine the impact of flare after DMARD-tapering on patients quantifying the changes in patient relevant domains e.g. functional ability, fatigue, quality of life, and worker productivity, and to explore the duration of the effect.

Methods: Data were used from all 113 patients that flared after tapering DMARDs in the TARA study, a multicenter randomised controlled trial in which patients in sustained remission $(\mathrm{DAS}<2.4 \& S J C<1$ ) tapered either their csDMARDs or TNF-inhibitor per protocol. For the current analysis, we compared the following secondary outcomes before and after flare over time; DAS44, functional ability (HAQ-DI), fatigue (BRAF-MDQ), quality of life (EQ-5D and SF36), anxiety and depression (HADS), morning stiffness, VAS general health, and worker productivity. Follow-up started three months before flare, at the moment of flare, and every 3 months thereafter. These were compared to baseline values, calculated by taking the average of 12, 9 and 6 months before flare. We used Linear Mixed Models (LMMs) with a random intercept and an autoregressive covariance matrix to determine an overall effect of flare. We adjusted for multiple testing with a Bonferroni correction. For determining the duration of the effect of a flare, we tested if the separate time points differed from the baseline with the same model. For the worker productivity we used descriptive statistics.

Results: In total, 113 patients experienced a flare. Patients who had a flare had a less stable course of the outcomes over time, compared with patients without flare (Figure 1A-G). When comparing all time points to baseline, statistical significant differences were found for DAS44 $(p<0.0001)$, VAS general health $(p<0.0001)$, morning stiffness $(p<0.0001)$, HAQ-DI $(p=0.0002)$, EQ-5D $(p<0.0001)$, BRAF-MDQ $(p=0.04)$, and the SF36 physical component scale $(\mathrm{p}=0.0004)$ (Figure $1 \mathrm{~A}-\mathrm{H})$. The duration of these effects was more than 12 months for the DAS44, morning stiffness, and VAS general health. For the HAQ-DI, EQ5D, BRAF-MDQ, and SF36 PCS the duration of the effect of a flare was 6 months (Figure $1 \mathrm{H})$. The effect sizes, expresses as differences with baseline, are indicated in Figure $1 \mathrm{H}$ and were rather small for most outcomes. The number of patients with payed work and unemployment were not affected by flare. The amount of sick leave increased from 1 to 1.3 days per month in the 6 months after flare and productivity decreased 1 point on a $0-10$ scale in the 3 months after flare.

Conclusion: Having a flare does affect patient reported outcomes. It affects DAS44, VAS general health, morning stiffness, functional ability, quality of life, and fatigue. This effect lasted for more than 6 months. Although we only found minor differences with baseline on group level, on the individual patient level this can still be of great importance.

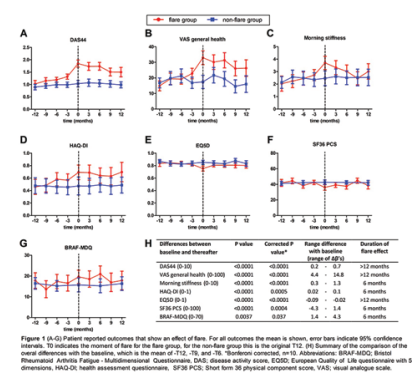

Disclosure of Interests: Elise van Mulligen: None declared, Angelique Weel: None declared, Martijn Kuijper: None declared, Johanna Hazes: None declared, Annette van der Helm - van Mil Grant/research support from: The research leading to these results has received funding from the European Research Council (ERC) under the European Union's Horizon 2020 research and innovation programme (Starting grant, agreement No 714312) and from the Dutch Arthritis Foundation.
The funding source had no role in the design and conduct of the study. Pascal de Jong: None declared

DOI: 10.1136/annrheumdis-2019-eular.4550

\section{SAT0116 DYNAMIC PREDICTION OF FLARES IN RHEUMATOID ARTHRITIS USING JOINT MODELLING AND MACHINE LEARNING: SIMULATION OF CLINICAL IMPACT WHEN USED AS DECISION AID IN A DISEASE ACTIVITY GUIDED DOSE REDUCTION STRATEGY}

Paco Welsing ${ }^{1}$, Alfons den Broeder ${ }^{2}$, Janneke Tekstra ${ }^{1}$, Marlies van der Goes ${ }^{1}$, Floris Lafeber ${ }^{1}$, Johannes W. G. Jacobs ${ }^{1}$, Oj Medina ${ }^{3}$, Asmir Vodencarevic ${ }^{4}$, Marcus Zimmermann-Rittereiser ${ }^{4}$, Saskia Haitjema ${ }^{3,5}$, Jacob M. van Laar ${ }^{1}$.

${ }^{1}$ University Medical Center Utrecht, Utrecht University, Utrecht, The Netherlands, Department of Rheumatology and Clinical Immunology, Utrecht, Netherlands; ${ }^{2}$ St. Maartenskliniek, Nijmegen, The Netherlands, Department of Rheumatology, Ubbergen, Netherlands; ${ }^{3}$ University Medical Center Utrecht, Utrecht University, Utrecht, The Netherlands, Applied Data Analytics in Medicine programme, Utrecht, Netherlands; ${ }^{4}$ Digital Services, Siemens Healthcare GmbH, Erlangen, Germany, Erlangen, Germany; ${ }^{5}$ University Medical Center Utrecht, Utrecht University, Utrecht, The Netherlands, Laboratory of Clinical Chemistry and Haematology, Utrecht, Netherlands

Background: Most rheumatoid arthritis (RA) patients on a (b)iological DMARD achieve and maintain remission or low disease activity. Then, tapering the drug is safe and cost-effective ${ }^{1}$, but with increased risk of short lived flares and joint damage progression. We developed so-called dynamic prediction models, using joint-modelling (JM) and machine learning (ML) respectively to predict an individual patients' risk of flaring These models can make short-term (e.g. 3 month) predictions repeatedly at every clinic visit, based on routine care data, i.e. the longitudinal course of the patients' disease activity and medication, demographic and disease characteristics. ${ }^{2}$

Objectives: To externally validate the $\mathrm{JM}$ and $\mathrm{ML}$ model and evaluate the clinical impact on flare occurrence and bDMARD use, when using predictions as decision aid for tapering bDMARDs in a protocolised disease activity guided tapering strategy.

Methods: For external validation, an RCT comparing protocolised dose reduction (stepwise increase injection interval, until flare or discontinuation, $n=121)$ with usual care $(n=59)$ was used.

Both models were applied to the trial data and AUC-ROC and calibration were assessed. To simulate our dose-reduction strategy, first, we defined optimal cut-offs for predictions using Youden's index as well as a cut-off at higher/lower predicted risks. Thereafter we applied them to the dose reduction arm of the trial. Assumptions were that 1) no further tapering was performed when the cut-off was reached without any flares occurring over time in the simulation and 2) when in the trial a flare occurred for which the bDMARD dose was increased to a value higher than when tapering was stopped in simulation, this flare occurred and the higher dose was used in simulation as well.

Results: AUC-ROC's in external validation were, as expected, somewhat lower than in the development cohort: $0.76(95 \% \mathrm{Cl} 0.71-0.85)$ and 0.73 (0.72-0.74) versus $0.78 / 0.79$ for JM/ML respectively, but still satisfactory. Calibration was better for ML, resulting in different cut-offs for the models. Results show that about $40 \%$ and $50 \%$ of flares can be prevented respectively and that the majority $(66 \%$ and $76 \%)$ of the dose reduction can be maintained using the optimal cut-offs in our prediction aided dose reduction strategy over 18 months. Table 1 shows the proportion of full dose used, mean number of flares per patient, and percentage of patients experiencing a flare for several cut-offs as well as in the disease activity guided dose reduction strategy (in bold). As context values are also shown for usual care (trial control arm; in italic).

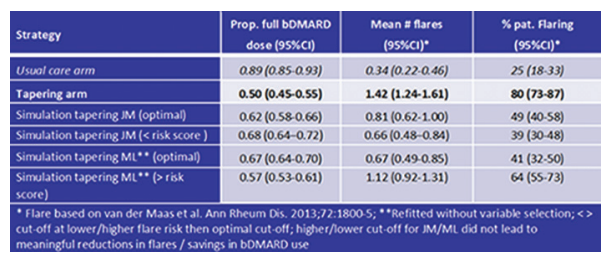

Conclusion: Both models proved to be externally valid. Using them to aid decisions on biological dose reduction has the potential to reduce the occurrence of flares significantly while largely retaining the reduction in dose as obtained by a disease activity guided dose reduction strategy. 